\title{
Effectiveness and Safety of Prenatal Phenobarbital for the Prevention of Neonatal Jaundice
}

\author{
T. VALAES, ${ }^{(40)}$ K. KIPOUROS, S. PETMEZAKI, M. SOLMAN, AND S. A. DOXIADIS \\ Institute of Child Health, Athens, Greece
}

\section{Summary}

The effect of $100 \mathrm{mg}$ of phenobarbital (PB) at bedtime for the last few wk of pregnancy on the incidence and severity of neonatal hyperbilirubinemia was studied. No effect was observed in the newborns of mothers who took less than ten tablets. In the 1310 newborns of adequately treated mothers $(P B \geq 1.0 \mathrm{~g})$, the incidence of marked jaundice (bilirubin $>16.0 \mathrm{mg} / \mathrm{dl}$ ) and the need to perform an exchange transfusion were reduced by a factor of six in relation to the incidence in 1553 control infants.

A randomly selected group of $\mathbf{4 1 5}$ children (182 control, $233 \mathrm{~PB}$ ) were reexamined at 61 to 82 months of age. There was no difference in the overall morbidity and mortality between the control and treatment group. A detailed neurologic assessment failed to reveal any differences between the two groups. In the VisuoMotor Integration test, the PB group scored significantly better than the control group. In the Draw-A-Woman and the Verbal Intelligence Test, the difference was in the same direction but was not statistically significant. The degree of jaundice was not found to significantly influence the performance in the neurological examination and the intelligence tests. Sensorineural hearing defect was significantly more common in the children with moderate or marked jaundice (bilirubin $>12 \mathrm{mg} / \mathrm{dl}$ ) than in those with lesser degrees of jaundice.

Prenatal PB is a practical, effective, and safe method for decreasing the incidence of neonatal hyperbilirubinemia.

\section{Speculation}

A wide variety of substances to which populations are exposed like alcohol and pesticides share with phenobarbital the ability to induce liver enzymes. It is possible that part of the variability in the ability of newborns to eliminate bilirubin is due to differences in exposure during fetal life to environmental inducing agents.

The ability of newborns to eliminate bilirubin is limited to a varying degree, and this is a major factor in the pathogenesis of neonatal unconjugated hyperbilirubinemia (8). Phenobarbital (PB) was found effective in decreasing the level of bilirubin in individuals with genetically determined decreased bilirubin conjugation $(2,6,16,37)$, and Trölle $(30)$, in a retrospective study, found diminished incidence of neonatal jaundice in infants of epileptic women treated with PB. Several studies demonstrated the effectiveness of prenatal $P B$ in reducing neonatal hyperbilirubinemia $(18,23,26,30,31)$. Because of the small number of subjects and the relative low risk of kernicterus in the populations studied, no proper assessment of the impact of such a preventive treatment on the overall problem of neonatal jaundice and its sequelae could be made. The present study provides this assessment in a large population of Greek newborns in which previous studies have shown an increased frequency of marked jaundice and kernicterus $(7,33)$.

Exposure to an agent during the fetal or newborn period invariably raises the spectrum of unsuspected long-term untoward effects. The possibity of long-term undesirable effects of PB cannot be denied on the basis that such effects have not been observed following therapeutic use of PB in the perinatal period. For this reason, 5 years after the completion of the initial study, a followup examination of the children exposed to prenatal PB was carried out with the aim of answering the question of the long-term effects of such a preventive treatment (36).

\section{MATERIALS AND METHODS}

\section{INITIAL STUDY}

Between October, 1968, and November, 1971, in two areas of Greece, the island of Lesbos and the city of Athens, pregnant women after informed consent participated in a controlled blind trial of PB. The methodology of the trial was described in detail elsewhere (35). In the treatment group, a tablet of $100 \mathrm{mg}$ PB was taken at bedtime for a variable period (maximum for $8 \mathrm{wk}$ ) depending on the interval between the prenatal visit at 34 to 36 wk of gestation and delivery. The control group received placebo tablets (Phase I). After the efficacy of PB and the absence of immediate untoward effects were demonstrated (35), all the women who attended for prenatal examination in their last 4 to 6 wk of pregnancy were given PB, whereas those who missed for one reason or another this prenatal examination were included in the control group (Phase II). This change in methodology was dictated by the need to provide an effective prevention of severe neonatal jaundice to this population experiencing both a high risk $(7,33)$ and considerable difficulties with the alternative treatment by exchange transfusion. It was realized that this change was going to affect the comparability of the two groups with an excess of women from rural areas and lower socioeconomic classes expected in the control group. Nevertheless, because the immediate aim was to assess the practical impact and to exclude uncommon complications of the treatment, both unlikely to be related to social class or place of domicile, we decided it was worthwhile to continue collecting data.

Cord blood was collected from all newborns for blood typing, direct Coombs test, and screening for G-6-PD deficiency (19). During Phase I, venous blood samples were drawn between 24 and $48 \mathrm{hr}$ after birth (first or second day of life) and on the fourth day of life. Consent for drawing one or both of these venous samples was refused on a few occasions. Bilirubin measurements were performed in all blood samples using either the method of Malloy and Evelyn (17) or the direct reading Bilirubinometer (American Optical Co., Buffalo, NY). Initial comparison as well as regular checking throughout the study period using "Versatol Pediatric" (Warner Lambert Co., Morris Plains, NJ) standard showed that the two methods gave comparable results.

During Phase II of the trial, no routine venous samples were drawn, but bilirubin measurements were done as frequently as required in infants with moderate or marked jaundice.

Throughout the study, the newborns were examined clinically daily, and the degree of jaundice was recorded. The physician responsible for this examination was unaware of whether the infant was in the control or PB group, and he was not responsible for the clinical management of the newborns. None of the infants 
was treated with phototherapy, and vitamin $\mathrm{K}$ was not given routinely at birth.

\section{FOLLOW-UP STUDY}

From the children of the original study, we chose to reexamine those living in the island of Lesbos as they constituted a relatively "captive" population with the additional advantage of small variation in socioeconomic and cultural conditions. For practical reasons, it was impossible to reexamine the total population. To secure an unbiased and informative sample, the following procedure was established. All the children of Phase I were designated for follow up. The children of Phase II were tabulated according to the family's place of residence which was classified as either urban, semirural, or rural, and the number of infants randomly selected from each area was proportional to the registered number of inhabitants. Finally, any child not included in the previous groups but with marked jaundice in the neonatal period was also designated to be reexamined.

After the list of children to be followed up was completed, the families were contacted by mail. Whenever there was no response, the local physicians, midwives, district nurses, or teachers were used to contact or obtain information about the family. Our team visited the towns and villages and examined the children in the local hospital, rural dispensary, or school. Those responsible for the examination had no access to the neonatal data.

The examination protocol included 137 precoded items and in many respects followed the protocol of the seventh year examination of the Perinatal Collaborative Project of the National Institute of Neurological and Communicative Disorders and Stroke (21). It included: (1) assessment of the socioeconomic class of the family on the basis of income, occupation, parental level of education, and quality of housing (29); (2) detailed general physical examination and medical and developmental history; (3) detailed neurologic examination with emphasis on fine motor coordination; (4) pure-tone audiometry; and (5) intelligence tests. Three tests were administered. The Visuo-Motor Integration (VMI) test (3) was used with the aim of assessing a function that has been reported to be particularly affected by hyperbilirubinemia. The Draw-a-Woman (DAW) test (11) was chosen as a test not depending on language. Finally, the Georgas Vocabulary (VOC) test (10) was included. All three tests have been standard. ized previously in a population of children living in Athens (10).

The data of the follow-up examination together with the neonatal data of each child were transferred to computer for storage and statistical analysis. Only the factors that were found to influence the outcome were included in the final regression equation of each dependent variable. Because prenatal phenobarbital significantly reduced neonatal bilirubin levels in all the least-squares multiple regression analyses, the variables of prenatal $P B$ versus control and that of degree of jaundice were introduced separately. In all the analyses involving the effect of hyperbilirubinemia, the children of phase I (routine bilirubin measurements) and those of phase II were analyzed separately, and only if similar results were obtained were they grouped together.

\section{RESULTS}

\section{EFFECT OF PRENATAL PB ON NEONATAL HYPERBILIRUBINEMIA}

In the two areas, 3075 newborns were included in the study, 2003 in Lesbos and 1072 in Athens, of which 1,522 were born to mothers who took PB. The infants of 212 mothers who took less than 10 tablets of PB (mean, 6.3 tablets), either because of a short interval between initiation of treatment and delivery or because of frequent omissions of the nightly tablet, showed no difference from the control group in either the mean bilirubin values (fourth day, $9.66 \pm 5.8 \mathrm{mg} / \mathrm{dl}$ ) or the incidence of marked jaundice. They are omitted from further analysis. There was no clear-cut relationship between number of tablets (beyond 10) and the degree of hyperbilirubinemia in the newborns.

Prenatal phenobarbital significantly lowered neonatal hyperbi- lirubinemia. In the group of term infants with no blood group incompatibility and normal G-6-PD activity in which bilirubin was measured routinely (phase I), a significant difference was present even in the cord blood (control, $1.69 \pm 0.57 \mathrm{mg} / \mathrm{dl}$; PB, $1.36 \pm 0.92 \mathrm{mg} / \mathrm{dl} ; P<0.001$ ), and by the fourth day of life, the mean bilirubin value of the control group $(9.45 \pm 5.43 \mathrm{mg} / \mathrm{dl})$ was double the value of the PB group $(4.76 \pm 4.06 \mathrm{mg} / \mathrm{dl} ; P<0.001)$.

Table 1 presents the incidence of marked jaundice (bilirubin $>16.0 \mathrm{mg} / \mathrm{dl}$ ) and exchange transfusion (performed when $\mathrm{BR} \geq$ $25 \mathrm{mg}$ in term infants without hemolytic disease). PB reduced the incidence of marked jaundice and the need for exchange transfusion among infants with nonspecific hyperbilirubinemia by a factor of six. In infants with ABO fetomaternal incompatibility, the incidence of marked jaundice was reduced by a factor of three whereas exchange transfusion was reduced by a factor of ten. All the three infants of the $\mathrm{PB}$ group that required exchange transfusion were from Lesbos and had ABO incompatibility. In addition, one was G-6-PD deficient, and the other had Down's syndrome and polycythemia. Altogether, there were 5 infants with Down's syndrome, and of these, 3 developed marked jaundice, and 2 required exchange transfusion.

More males than females developed marked jaundice and required exchange transfusion, but this difference did not reach a level of statistical significance. In the control group, the incidence of exchange transfusion was significantly higher $(P<0.05)$ in Lesbos than in Athens for infants with nonspecific hyperbilirubinemia as well as for those with $\mathrm{ABO}$ incompatibility. No difference was found in the incidence of jaundice in relation to the rural or urban place of domicile in the island of Lesbos.

In the total material of 3075 newborns, there were two infants with hemorrhagic manifestations. One developed a massive subgalleal cephalohematoma following forceps delivery, and the other presented with hematemesis and melena. Both infants recovered after transfusion and vitamin $K$. Both infants belonged to the PB group. These two cases were the only ones in which PB administration to the mother could be incriminated for a complication in the newborn. With the dose of PB used in the trial, no infant with symptoms suggesting withdrawal was observed. Sleeping and feeding patterns were not different in the infants of the control and $\mathrm{PB}$ group. None of the infants with marked jaundice showed signs of bilirubin encephalopathy in spite of bilirubin values in excess of $25 \mathrm{mg} / \mathrm{dl}$ in some and a delay of several $\mathrm{hr}$ for the infants from Lesbos to be transported to Athens for exchange transfusion. The complications for the mothers were insignificant. Only two mothers developed a rash, and eight mothers dropped out of the trial because they did not tolerate the morning drowsiness common in the first 3 to 4 days of the treatment.

\section{FOLLOW-UP EXAMINATION OF THE CHILDREN EXPOSED PRENATALLY TO PB}

From the total group of 2003 infants from Lesbos, 719 (36\%) were designated for follow up, and of these, 490 were actually reexamined. The reason for which 229 children were not examined were individually scrutinized and included: moving out of the island (103), inability to trace the family (28), death of the children (6 in the PB group and 7 in the control), and refusal ( 1 in the PB group and 4 in the control). Finally, in 80 cases, the family was contacted, but we were not able to examine the children for such reasons as time limitations, absence of the family, etc. We have no reason to suspect that our inability to examine any of these 229 children had any relation to the outcome.

Of the 490 children reexamined, 75 were removed from the final analysis for the following specific reasons: preterm delivery (17), low birth weight (5), multiple pregnancy (8), PB dose $<1.0$ g (35), incomplete examination (4), and definite clinical diagnoses known to affect somatic and mental development (6). Thus, 415 term, singleton with birth weight $>2.5 \mathrm{~kg}$ are included in the final analysis. Of the 415 children, 182 belonged to the control, and 233 belonged to the PB group. At the time of the examination, the age of the children varied between 61 and 82 months.

The control and the prenatal PB groups differed significantly 
Table 1. Incidence of marked jaundice and exchange transfusions in the control and treatment groups.

\begin{tabular}{|c|c|c|c|c|c|c|}
\hline \multirow{2}{*}{ Diagnostic categories } & \multicolumn{3}{|c|}{ Control } & \multicolumn{3}{|c|}{ Phenobarbital $\geq \mathrm{lg}$} \\
\hline & Total & Marked jaundice & $\begin{array}{l}\text { Exchange transfu- } \\
\text { sion }\end{array}$ & Total & Marked jaundice & $\begin{array}{l}\text { Exchange transfu- } \\
\text { sion }\end{array}$ \\
\hline Nonspecific hyperbilirubinemia & 1181 & $66 \quad(5.5 \%)$ & $9(0.76 \%)$ & 820 & $5(0.6 \%)$ & 0 \\
\hline $\mathrm{ABO}$ incompatibility & 204 & $21(10.2 \%)$ & $12(5.8 \%)$ & 168 & $6(3.5 \%)$ & $1(0.59 \%)$ \\
\hline G-6-PD deficiency & 78 & $11(14.2 \%)$ & 0 & 61 & $2(3.2 \%)$ & $1(1.6 \%)$ \\
\hline $\begin{array}{l}\text { Other and insufficient informa- } \\
\text { tion }\end{array}$ & 90 & $4(4.4 \%)$ & $1(1.1 \%)$ & 261 & $2(0.7 \%)$ & $1(0.3 \%)$ \\
\hline Total & 1553 & $102(6.6 \%)$ & $22(1.4 \%)$ & 1310 & $15(1.1 \%)$ & $3(0.23 \%)$ \\
\hline
\end{tabular}

$(P<0.001)$ in relation to place of residence (excess of urbanites in the PB group), social class (control: low, 145; middle, 33; high, 4. PB: low, 133; middle, 92; high, 8), age distribution (excess of older children in the control group), and, of course, incidence of neonatal jaundice. There were almost equal number of males and females in both groups.

As assessed from the history and physical examination, the children were remarkably healthy with upper respiratory infections the commonest complaint by history and serous otitis media by examination. One child in each group was affected by chronic asthmatic bronchitis. Also, two children, one in each group, had grand mal seizures. In both cases, the perinatal history was unremarkable. There were 7 children in each group with a history of febrile convulsions.

Physical Growth. In physical growth the sex, the social class, and the place of residence were found to be important variables particularly for height (Table 2). Beyond the effect of these factors, there appears to be an advantage conferred by PB which reached level of statistical significance only for height.

Neurologic Evaluation. There were two children, one in each group, with well-defined neurologic abnormality. The one belonging to the PB group was hyperkinetic and had increased deep tendon reflexes and poor coordination but performed at an average level in the intelligence tests. His perinatal and subsequent medical history were unremarkable. The other child had increased deep tendon reflexes and hypertonicity in all extremities and fine tremor of the upper extremities. Again, apart from mild jaundice, his history was unremarkable.

In addition to the classical neurologic examination, the tests described by Touwen and Prechtl (28) were used to detect minor degrees of neurologic dysfunction and assess the degree of neurologic maturation. Apart from the two children already described with a well-defined neurologic abnormality, the rest functioned normally. Many of the children did not receive optimal scores in every test, but no constant pattern of abnormalities was recognized. As for the significance of nonoptimal scores, we felt that a straight qualitative judgment of normal or abnormal was arbitrary, and in most cases, it would not represent the overall performance of the children. We choose to present this part of the neurologic examination in a quantitative way. For the following tests in which a high proportion of children performed nonoptimally, the scores were added to give a cumulative score, coordination score: (1) associated movements during walking on tiptoe and on the heels; (2) standing with eyes closed; (3) mouth opening-finger spreading phenomenon; (4) diadochokinesis and associated movements; (5) finger-nose test; (6) finger opposition test; (7) knee-heel test. In scoring the tests, the optimal performance was given a score of 1 , and the least adequate performance was given a score of 3 or 4 . Right and left sides were scored separately. Thus, the cumulative score could vary from 22 to 74 .

This score, as expected, was found to be significantly related to age $(P<0.001)$ and sex $(P<0.02)$, the girls scoring better than the boys. The social class, the place of residence, the degree of
Table 2. Significance of factors affecting physical growth

\begin{tabular}{lll}
\hline \multicolumn{1}{c}{ Measurements } & \multicolumn{1}{c}{ Factors } & $P^{1}$ \\
\hline Height & Males $>$ females & $<0.01$ \\
& Urban $>$ rural & $<0.001$ \\
& Social class: high $>$ low & $<0.02$ \\
& PB $>$ control & $<0.02$ \\
& & \\
Meight & Males $>$ females & $<0.2$ \\
& Social class: high $>$ low & $<0.01$ \\
& PB $>$ control & $<0.1$ \\
& & $<0.2$ \\
Head circumfer- & Males $>$ females & $<0.001$ \\
ence & Urban $<$ rural & $<0.2$ \\
& Social class: high $>$ low & $<0.2$ \\
& PB $>$ control & $<0.1$ \\
\hline
\end{tabular}

${ }^{1}$ Based on least-squares multiple regression analysis.

neonatal jaundice, and the prenatal administration of $\mathrm{PB}$ did not influence the score significantly.

Hearing. Pure-tone audiometry revealed various degrees of hearing loss in 39 children. Conductive hearing defect was present in 29 children (control group, 13, 7.14\%; PB, 16, 6.8\%).

In 10 children, sensorineural hearing defect was diagnosed. In Table 3, this group is analyzed in relation to the degree of neonatal jaundice. Sensorineural hearing defect was significantly more common in the children with moderate or marked neonatal jaundice $(9.2 \%)$ than in the children with slight or no jaundice $(1.1 \%)$. In only two children, both with marked jaundice; the hearing defect impaired their performance in the verbal intelligence test.

Intelligence. When the raw scores in the three intelligence tests were transformed to intelligence quotients on the basis of the standardization in Athenian children (10), the distribution of IQ's in our population had a mode and mean in the range of 80 to 90 for all three tests. This raised doubts on the validity of the standardization for the children in the island of Lesbos. We resolved this problem by using for our analysis the raw scores.

In the multiple regression analysis sex, place of residence, and social class were found to be important to a variable extent (Table 4). Females scored significantly better in the DAW test. The difference in the other two tests was not statistically significant. In the VMI and DAW tests, children of towns scored better than did children of villages. Surprisingly, the difference in the verbal test, although in the same direction, was not statistically significant. We suspect that the difference in kindergarden attendance between the urban and rural populations (almost 100\% of the children in the town of Mytilene attended kindergarden, the attendance was $54 \%$ in the semirural areas, and only $8 \%$ in the rural villages) exaggerated the difference in the two tests that relied on dexterity in the use of pencil and in drawing. In the VMI and VOC tests, children of the higher social classes scored signifi- 
Table 3. Sensorineural hearing loss in relation to the degree of neonatal jaundice

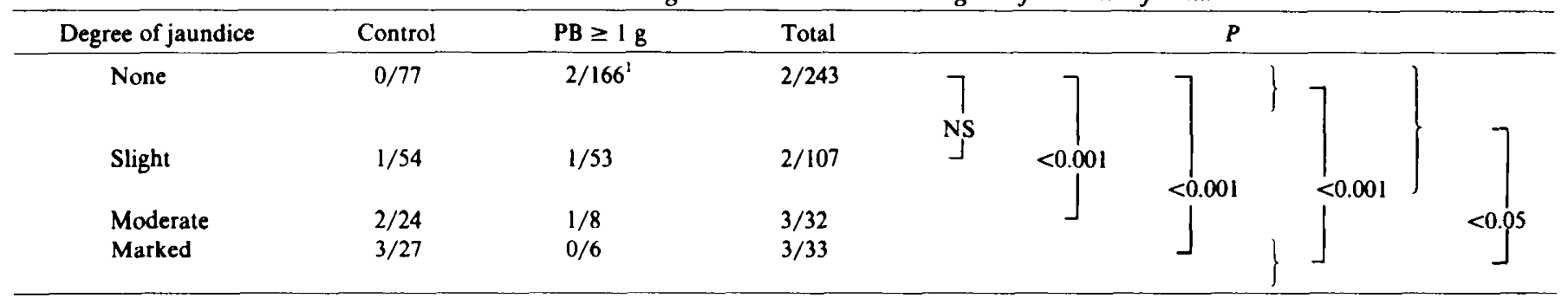

${ }^{1}$ Number with sensorineural hearing loss/total number in group.

Table 4. Significance of factors affecting performance in intelligence tests

\begin{tabular}{cll}
\multicolumn{1}{c}{ Factors } & \multicolumn{1}{c}{$\boldsymbol{P}^{\prime}$} \\
\hline Tests & \multicolumn{1}{c}{ Males $>$ females } & $<0.1$ \\
VMI & Urban $>$ rural & $<0.001$ \\
& Social class: high $>$ low & $<0.020$ \\
& PB $>$ control & $<0.025$ \\
& (No jaundice $>$ jaundice) & $<0.1$ \\
& Males $<$ females & $<0.6$ \\
VOC & Urban $>$ rural & $<0.1$ \\
& Social class: high $>$ low & $<0.001$ \\
& PB $>$ control & $<0.9$ \\
& (No jaundice $>$ jaundice) & $<0.4$ \\
& Males $<$ females & $<0.001$ \\
DAW & Urban $>$ rural & $<0.001$ \\
& Social class: high $>$ low & $<0.2$ \\
& PB $>$ control & $<0.2$ \\
& (No jaundice $>$ jaundice) & $<0.9$ \\
\hline
\end{tabular}

'Based on least-squares multiple regression analysis. In the equations, the variables of degree of jaundice and of treatment or control groups were introduced separately.

cantly better than did children of the lower classes. In the DAW test. the difference was in the same direction but did not reach statistically significant level. In all three tests, the PB group scored better than did the control, but only in the VMI test was the difference statistically significant. Finally, the degree of jaundice did not effect significantly any of the tests.

There was a highly significant $(P<0.001)$ correlation between the three intelligence tests. (DAW versus VMI: $r=0.79$; VMI versus VOC: $r=0.59$; VOC versus DAW: $r=0.65$ ). Similarly, there was a significant $(P<0.001)$ correlation between the VMI and coordination score $(r=0.55)$.

Children with Marked Neonatal Jaundice. The degree of jaundice was not found to be an important factor in either the neurologic outcome or the intelligence tests scores. Inasmuch as bilirubindependent central nervous system damage might not be produced in a continuous manner at all bilirubin levels but may be the result of bilirubin exceeding a threshold level, the performance of children with marked neonatal jaundice (peak bilirubin $>16 \mathrm{mg} / \mathrm{dl}$ ) was examined. In this analysis, apart from the 33 infants with marked jaundice belonging to the group of 415 infants, we included 12 infants with marked jaundice belonging to the 75 infants excluded so far from the analysis. (One infant with Down's syndrome and marked jaundice was not included because formal intelligence testing was not performed.)

In Table 5, the main data on the 44 infants with marked jaundice are presented. From Figures 1 and 2 , it is obvious that the children with marked neonatal jaundice did not differ from the rest of the population as far as their performance was concerned. Similarly, within the group with marked jaundice, those with the higher bilirubin levels did not differ from the rest. Two children with marked hearing loss in the high frequencies, mentioned already, scored low in the verbal test, but their performance in the other tests was not different from the rest of the population.
Table 5. Analysis of children with marked neonatal jaundice

\begin{tabular}{lcccc}
\hline \multirow{2}{*}{ Diagnostic categories } & \multicolumn{3}{c}{ Peak bilirubin (mg/dl) } & \multirow{2}{*}{ Total } \\
\cline { 2 - 4 } & $16-20$ & $20.1-25$ & $>25$ & \\
\hline ABO incompatibility & 4 & $4(1)^{1}$ & $5(5)$ & $13(6)$ \\
$\begin{array}{l}\text { Nonspecific hyperbilirubi- } \\
\quad \text { nemia }\end{array}$ & 16 & 4 & $4(2)$ & $24(2)$ \\
$\begin{array}{l}\text { G-6-PD deficiency } \\
\text { Prematurity }\end{array}$ & & 1 & & \\
Total & 1 & 4 & $2(1)$ & $7(1)$ \\
& 21 & $13(1)$ & $11(8)$ & $45(9)$ \\
\hline
\end{tabular}

${ }^{1}$ Numbers in parentheses, number of children exchange transfused.

${ }^{2}$ Gestational age, 33 to $36 \mathrm{wk}$; birth weight, 2100 to $3000 \mathrm{~g}$. In one infant, $A B O$ incompatibility and in another G-6-PD deficiency were also present.

These results are in agreement with the fact that in the newborn period none of the children demonstrated signs or symptoms of bilirubin encephalopathy.

\section{DISCUSSION}

The results of the present as well as of similar trials $(18,23,26$, 31 ) indicate that adequate prenatal PB treatment significantly decreases the problem of neonatal jaundice. In the group of newborns whose mothers received at least $10(100 \mathrm{mg}$ PB) tablets, the incidences of marked jaundice and exchange transfusion was reduced by a factor of six. These clinical results should be ascribed to the action of PB on the liver enzymatic mechanism of bilirubin elimination $(5,8)$. This effect can best be described in clinical terms as an increase in the proportion of neonates born with an adequate mechanism for handling bilirubin.

In the present material, it appears that there was no benefit for the infants of mothers who received less than $1.0 \mathrm{~g}$ of PB in 100 $\mathrm{mg}$ doses at bedtime. Similarly, smaller daily doses $(30 \mathrm{mg})$, were not found as effective even when treatment was prolonged for as many as $8 \mathrm{wk}(12,27)$. This observation together with the demonstration of a dose-effect relationship in postnatal treatment of neonatal jaundice with PB (32) indicate that dosage of PB is important together with the duration of treatment for the induction of the enzymatic mechanism. The lack of clear association in our material between total maternal PB dose (above $1.0 \mathrm{~g}$ ) and bilirubin levels in the newborn may reflect the fact that the clinically measurable endpoint, e.g., the bilirubin level, is only partially determined by bilirubin elimination, the function influenced by PB. For all these reasons, the minimum dose and length of administration that will produce an appreciable clinical effect cannot be considered as established.

In our study, as well as in other studies $(26,31)$, a small percentage of the infants apparently failed to benefit from the prenatal PB treatment. In the absence of the relevant measurements, we cannot decide between the various possible explanations: (1) inadequate response; (2) inadequate blood levels of PB, and (3) excessive hemolysis exceeding the capacity of even an adequately activated bilirubin-eliminating system.

Neither our own nor other studies has provided evidence on the gestational age at which the effect of PB can be obtained $(9,32)$. An equally important unanswered question is whether the biliru- 

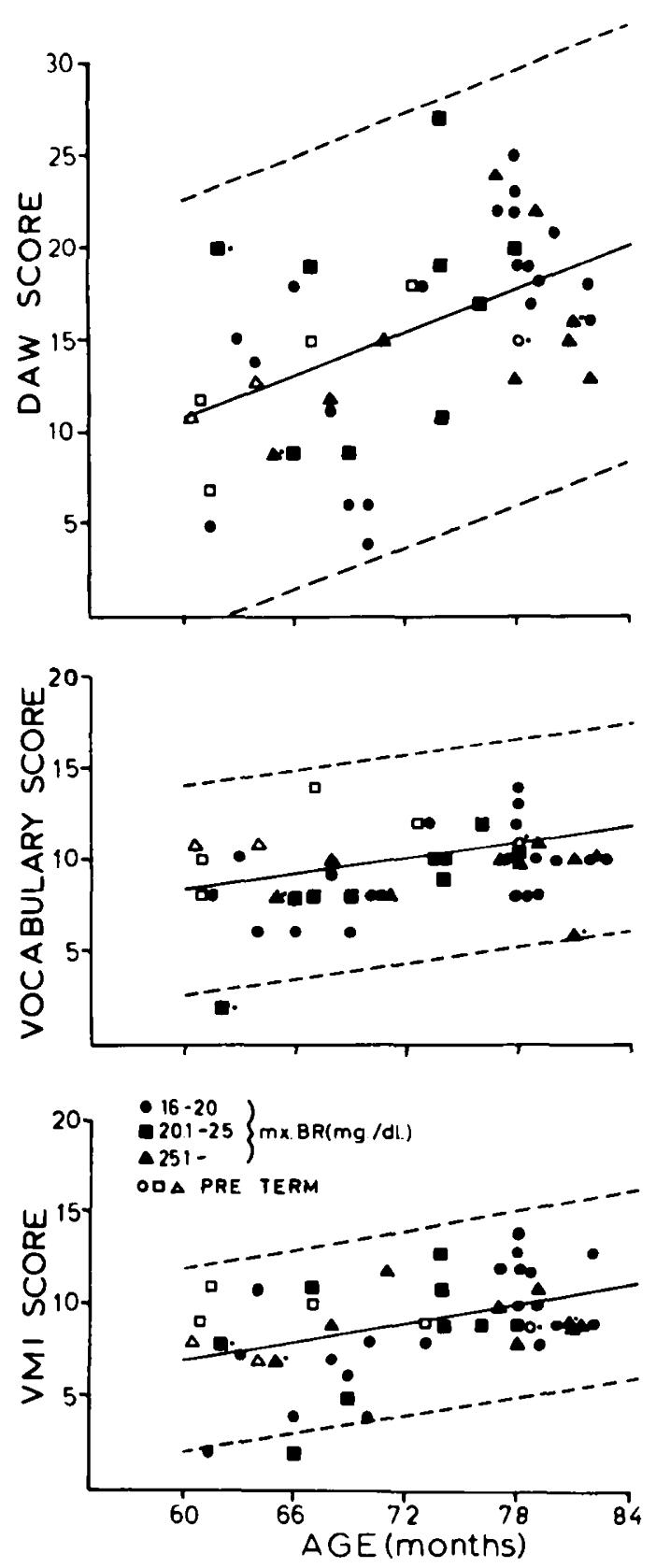

Fig. 1. Individual raw scores in three intelligence tests of children with marked neonatal jaundice. Dots on the right side of symbols, children with sensorineural hearing defect. Regression line and $95 \%$ confidence limits calculated from the scores of the total material of 415 children.

bin-eliminating mechanism will continue at the same level of activity if it has been induced by PB and the drug is discontinued while delivery is delayed. Obviously, the answer to these questions will enable us to devise a scheme of treatment that will expose the fetus at the right time and just enough for the best practical results.

The financial benefits from the prenatal PB, although not analyzed systematically, were considerable, particularly for the infants in the island of Lesbos who had to be air transported to Athens for the management of marked jaundice. Against the expenses for the monitoring and treatment of marked jaundice, the cost of prevention was only a small fraction. For the entire trial, the wholesale cost for the PB and placebo tablets was equivalent to the cost of one exchange transfusion.

Previous reports indicated that anticonvulsant treatment during pregnancy increased the incidence of severity of hemorrhagic disease of the newborn $(1,15,20)$. Although our mothers were exposed to a small dose of PB and only for a few wk, towards the

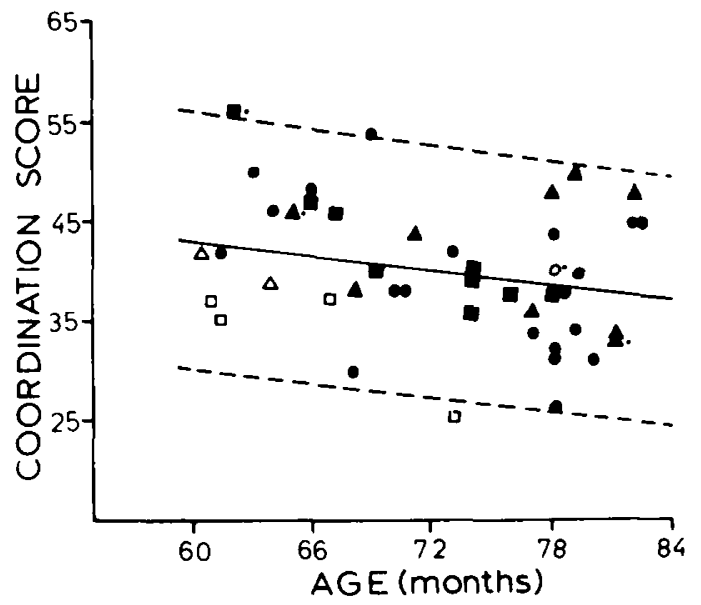

Fig. 2. Individual coordination scores (cumulative score of 11 neurological tests assessing motor coordination and maturation) in children with marked jaundice. Code as in Figure 1. Optimal performance gives low score.

end of pregnancy decreased levels of clotting factors were observed in their newborns (34). Hemorrhagic manifestations were observed in only two infants. It is likely that even these two cases would have been prevented if vitamin $\mathbf{K}$ was given routinely to our newborns. This is suggested by the fact that in the infants who received vitamin $\mathbf{K}$ the coagulation tests were promptly normalized (34).

In relation to the long-term effects of prenatal $\mathrm{PB}$, the results of our follow up are reassuring. There was no difference in mortality and morbidity between the control and treatment groups and no suggestion of differences in susceptibility to infections, to seizures, and to neurologic abnormalities. The overall low incidence of overt neurologic abnormalities is not surprising in view of the fact that our material consisted of term, appropriate for gestational age babies with benign perinatal and subsequent histories.

In physical growth, the coordination score and the intelligence tests prenatal PB exposure conferred an advantage which reached statistical significance only for height and the VMI test. Inasmuch as the control and PB groups differed in two of the factors (social class and place of residence) that were expected to influence the outcome, we relied on the statistical technique of multiple regression analysis to separate the effects of these factors from that of the treatment and the resultant difference in the incidence of jaundice. Confidence in these results is dependent on the power of the statistical method to separate the effects of the different factors on the outcome. For the purpose of our study, suffice it to say that these results make it highly unlikely that PB had longterm untoward effects in growth and development that were not uncovered by our method of analysis.

Any long-term beneficial effects of prenatal PB were expected to be related to the decrease in the level of neonatal hyperbilirubinemia. Exchange transfusion was used in our population, and no case of bilirubin encephalopathy was suspected in the neonatal period. Nevertheless, it has been suggested that subtle degrees of brain damage result from bilirubin levels not considered high enough to warrant treatment $(13,22,24)$.

With the exception of sensorineural hearing loss, the degree of neonatal jaundice was not significantly related to the outcome. Even when the performance of the children with marked neonatal jaundice was compared with the rest of the population no difference became apparent. It should be emphasized again that in spite of high bilirubin levels, in none of these children was kernicterus suspected in the neonatal period.

Sensorineural hearing loss was more common in children who had moderate (bilirubin, $>12 \mathrm{mg} / \mathrm{dl}$ ) or marked neonatal jaundice than in those with lesser levels of hyperbilirubinemia. Even this group of infants, with evidence of selective nervous system damage from bilirubin, performed adequately in the intelligence tests and 
in motor coordination. Thus our follow-up examination failed to reveal evidence for an extended spectrum of long-term sequelae of neonatal hyperbilirubinemia beyond the well-recognized kernicterus and hearing loss. Whether kernicterus can occur with levels of bilirubin below $20 \mathrm{mg} / \mathrm{dl}$ is a separate question. Assessment of bilirubin binding in newborn plasma demonstrated that a variety of conditions (prematurity, acidosis, and drugs) are associated with significant amounts of loosely bound bilirubin with relatively low levels of unconjugated bilirubin $(4,14)$. Under these conditions, kernicterus has occurred with indirect bilirubin levels as low as 8 to $12 \mathrm{mg} / \mathrm{dl}(8,25)$. Because such predisposing factors were absent in our material, adherence to standard criteria for the performance of exchange transfusion was sufficient to prevent bilirubin encephalopathy. Nevertheless, we have to accept that hearing loss, as a result of bilirubin toxicity, was not totally prevented.

\section{CONCLUSION}

Because of high incidence, serious sequelae, complexity, and cost of treatment, neonatal jaundice is a candidate for preventive treatment. Such a treatment has to be efficient, simple, unexpensive, and without important immediate and long-term untoward effects. Our studies have demonstrated that prenatal PB fulfills all the above conditions. As with all preventive methods, a large number had to be treated to prevent significant risk in a few. One hundred pregnant women had to be treated to prevent dangerous levels of bilirubin in five of their infants. The high incidence of marked jaundice and the need for infants from Lesbos to be transferred to Athens for the management of marked jaundice easily make the advantages of prevention outweigh the disadvantages. Whether this preventive treatment should be applied to all pregnant women or only to special high-risk groups should be decided on the basis of incidence of marked jaundice in a given population and the availability, risk, and cost of other methods of managment.

\section{REFERENCES AND NOTES}

I. Alagille, D., Odievre, M., Houllemare, L., Viterbo, G., and Leroy, D.: Avitaminose $K$ neonatale severe chez 2 enfants nès de mère traité par des anticomitiaux. Arch. Fr. Pediatr., 25: 31 (1968).

2. Arias. 1.. Garner. L. M.. Cohen, M.. BenEzzer, Y., and Levi, A. J.: Chronic nonhemolytic unconjugated hyperbilirubinemia with glucuronyl transferase deficiency. Am. J. Med.. 47: 395 (1969).

3. Beery, K. E., and Buktenica, N. A.: Developmental test of visual-motor integration. (Follett Educational Corporation. Chicago. 1967).

4. Cashore. W. J., Horwich. A., Karotkin, E. H., and Oh, W.: Influence of gestational age and clinical status on bilirubin-binding capacity in newborn infants. Am. J. Dis. Child., I31: 898 (1977).

5. Catz. C.. and Yaffé, S. J.: Barbiturate enhancement of bilirubin conjugation and excretion in young and adult animals. Pediatr. Res., 2: 361 (1968).

6. Crigler. J. F., and Gold, N. I.: Sodium phenobarbital-induced decrease in serum bilirubin in an infant with congenital nonhemolytic jaundice and kernicterus. J. Clin. Invest., 45: 998 (1966).

7. Doxiadis, S. A.. Valaes. T.. Karaklis, A., and Stavrakakis, D.: Risk of severe jaundice in glucose-6-phosphate dehydrogenase deficiency of the newborn Differences in population groups. Lancet, 2: 1210 (1964).

8. Gartner, L. M., Lee. K. S., Vaisman. S., Lane. D., and Zarafu, I.: Development of bilirubin transport and metabolism in the newborn thesus monkey. $J$ Pediatr., 90: 513 (1977).

9. Gartner. L. M., Snyder. R. N., Chadon. R. S., and Bernstein. J.: Kernicterus: high incidence in premature infants with low serum bilirubin concentrations. Pediatrics, 45: 906 (1970).

10. Georgas. J.: The Georgas Intelligence Test for children. (Kedros, Athens, 1971).

11. Goodenough, F. L.: Measurement of intelligence by drawings. (World Book Co. Chicago. 1926).

12. Halpin, T. F., Jones, A. R., Bishop, H. L.. and Lerner. S.: Prophylaxis of neonatal hyperbilirubinemia with phenobarbital. Obstet. Gynecol., 40: 85 (1972).

13. Johnson, L.., and Boggs, T. R.: Bilirubin-dependent brain damage: incidence and indications for treatment. In: B. G. Odell, R. Schaffer, A. P. Simopoulos:
Phototherapy in the Newborn: an Overview. p. 122 (National Academy of Sciences, Washington, D. C.. 1974).

14. Kapitulnik, J., Valaes, T., Kaufmann, N. A., and Blondheim, S. H.: Clinical evaluation of Sephadex gel filtration in estimation of bilitubin binding in serum in neonatal jaundice. Arch. Dis. Child., 49: 886 (1974).

15. Kohler, H. G.: Haemorrhage in the newborn of epileptic mothers. Lancet, $1: 267$ (1966).

16. Kreek. M. J.. and Sleisenger, M. H.: Reduction of serum-unconjugated-bilirubin with phenobarbitone in adult congenital nonhaemolytic unconjugated hyperbilirubinemia. Lancet, 2: 73 (1968).

17. Malloy, H. T., and Evelyn, K. A.: The determination of bilirubin with the photoelectric colorimeter. J. Biol. Chem., 119:481 (1937).

18. Maurer, H. M., Wolfr. J. A., Finster, M., Poppers, P. J., Pantuck, E., Kuntzman, R., and Conney, A. H.: Reduction in concentration of total serum-bilirubin in offspring of women treated with phenobarbitone during pregnancy. Lancet, 2 : 122 (1968).

19. Motulsky, A. G., and Campbell-Kraut, J. M.: Population genetics of glucose-6phosphate dehydrogenase deficiency of the red cell. In: B. S. Blumberg: Proceedings of the Conference on Genetic Polymorphism and Geographic Variations in Disease. p. 159 (Grune \& Stratton, New York, 1961).

20. Mountain, K. R., Hirsh, J., and Gallus, A. J.: Neonatal coagulation defect due to anticonvulsant drug treatment in pregnancy. Lancet, $1: 265$ (197).

21. Niswander, K. R., and Jordan, M. (Eds.): The collaborative perinatal study of NINDB. (W. B. Saunders Co., Philadelphia, 1972).

22. Odell, G. B., Storey, G. N. B., and Rosenberg, L. A.: Studies in kernicterus. III. The saturation of serum proteins with bilirubin during neonatal life and its relationship to brain damage at five years. J. Pediatr., 76: 12 (1970).

23. Rainboer, C.. Thomson, R. P. H., and Williams, R.: Controlled trials of phenobarbitone therapy in neonatal juandice. Lancet, 1: 966 (1969).

24. Scheit, P. C., Mellits, E. D., Hardy, J. B., Drage, J. S., and Boggs, T. R.: Toxicity to bilirubin in neonates: infant development during first year in relation to maximum neonatal serum bilirubin concentrations. J. Pediatr., 91: 293 (1977).

25. Stern, L., and Denton, R.: Kernicterus in small premature infants. Pediatrics, 35 : 483 (1965).

26. Thomas, C. R.: Routine phenobarbital for prevention of neonatal hyperbilinubinemia. Obstet. Gynecol., 47: 304 (1976).

27. Thomas, C. R.: Phenobarbital prophylaxis of neonatal hyperbilirubinemia. Obstet. Gynecol., 50: 607 (1977).

28. Touwen, B. C. L., and Prechtl. H. F. R.: The neurological examination of the child with minor nervous dysfunction. Clinics in Developmental Medicine. Vol. 38 (Heinemann, London, 1971).

29. Triandis. H., Vassiliou. V., and Thomanek, E. K.: Social status as a determinant of respect and friendship acceptance. Sociometry. 29: 396 (1966).

30. Trölle, D.: Phenobarbitone and neonatal icterus. Lancet, 1 : 251 (1968).

31. Trölle, D.: Decrease of total serum bilirubin concentration in newborn infants after phenobarbitone treatment. Lancet, 2: 705 (1968).

32. Vaisman, S. L., and Gartner, L. M.: Pharmacologic treatment of neonatal hyperbilinubinemia. Clin. Perinatol.. 2: 37 (1975).

33. Valaes. T., Karaklis. A.. Stavrakakis. D.. Bavela-Stavrakakis. K.. Perakis, A.. and Doxiadis, $S$. A.: Incidence and mechanisms of neonatal jaundice related to $G$ 6-PD deficiency. Pediatr. Res., 3: 448 (1969).

34. Valaes. T., and Petmezaki, S.: Coagulation abnormalities in the neonate after prenatal phenobarbital exposure. Iatriki. 24: 325 (1973).

35. Valaes. T.. Petmezaki, S., and Doxiadis, S. A.: Effect on neonatal hyperbilirubinemia of phenobarbital during pregnancy or after birth: practical value of the treatment in a population with high risk of unexplained severe neonatal jaundice. In: Bilirubin Metabolism in the Newborn. Birth Defects, Original Article Series. The National Foundation, March of Dimes. 6: 46 (1970)

36. Wilson. J. T.: Phenobarbital in the perinatal period. Pediatrics, 43: 324 (1969).

37. Yaffé, S. J., Levy, G., Matsuzawa, T., and Baliah. T.: Enhancement of glucuronide conjugating capacity in a hyperbilirubinemic infant due to apparent enzymic induction by phenobarbital. N. Engl. J. Med., 275: 1461 (1966).

38. A detailed description of the process of selection of the follow-up group, details on those not examined and those excluded from the analyses, as well as full results of the multiple regression analyses will be sent to interested readers on request.

39. The authors thank the obstetricians and nursing staff of the maternity units in the island of Lesbos and the "Metera" maternity hospital in Athens for their enthusiastic collaboration. The Civil and Health authorities of Lesbos were extremely helpful for tracing the families. We are particularly grateful to $\mathrm{K}$. Adreopoulos for coordinating this aspect of the project. We express our appreciation to Soula Zografou for the meticulous care with which she performed all the laboratory determinations, to $S$. Agellopoulos, biostatistician, for her help in selecting the sample for the follow-up, to D. Micheologiannis, M.D. for designing the neurological examination, and to S. Petmezaki. M.D. for his expert help with the audiometry.

40. Requests for reprints should be addressed to: Timos Valaes. M.D.. Department of Pediatrics, Tufts-New England Medical Center Hospital, 171 Harrison Avenue, Boston, MA 02111 (USA).

41. Received for publication April 2, 1979

42. Accepted for publication December 11, 1979. 